\title{
MicroRNA-107 Targets IKBKG and Sensitizes A549 Cells to Parthenolide
}

\author{
SOKVISETH MOENG ${ }^{1 *}$, HYUN AH SEO $^{1 *}$, CHO YEAN HWANG $^{1 *}$, GABRIEL A. CIPOLLA $^{2}$, \\ DONG JIN LEE ${ }^{3}$, HYO JEONG KUH ${ }^{4}$ and JONG KOOK PARK ${ }^{1}$ \\ ${ }^{I}$ Department of Biomedical Science, Hallym University, Chuncheon, Republic of Korea, \\ ${ }^{2}$ Department of Genetics, Federal University of Paraná, Curitiba, Brazil; \\ ${ }^{3}$ Department of Otorhinolaryngology-Head and Neck Surgery, \\ Hallym University College of Medicine, Seoul, Republic of Korea; \\ ${ }^{4}$ Department of Medical Life Sciences, College of Medicine, \\ The Catholic University of Korea, Seoul, Republic of Korea
}

\begin{abstract}
Background/Aim: Patients with advanced non-small cell lung cancer (NSCLC) frequently face a dismal prognosis because of lack of curative therapies. We, therefore, conducted a preclinical investigation of the therapeutic efficacy of microRNA-107 (miR-107). Materials and Methods: The effects of miR-107 on cell proliferation and target gene expression were studied. Combinatorial effects of miR-107 and parthenolide were evaluated. Results: Cell proliferation was repressed in A549 NSCLC cells transfected with miR-107. Inhibitor of nuclear factor kappa B kinase subunit gamma was directly targeted by miR-107. Overexpression of miR-107 in A549 cells sensitized them to parthenolide along with a marked reduction of cyclindependent kinase 2. Conclusion: Our findings unveil an important biological function of miR-107 in regulating lung cancer cell proliferation and elevating an antiproliferative effect of parthenolide on lung cancer cells, suggesting that miR-107 could be beneficial benefit treatment for advanced NSCLC.
\end{abstract}

Lung cancer is one of the leading causes of cancer-related deaths worldwide, with non-small cell lung cancer (NSCLC) accounting for approximately $80 \%$ of lung cancer cases (1). Radical surgery has been recommended as a standard treatment for early-stage NSCLC (2). Other treatments for

\footnotetext{
*These Authors contributed equally to this work.

Correspondence to: Jong Kook Park, Ph.D., Department of Biomedical Science and Research Institute for Bioscience \& Biotechnology, Hallym University, Chuncheon 24252, Republic of Korea. Tel: +82 332482114, Fax: +82 332563420, e-mail: jkp555@hallym.ac.kr
}

Key Words: MicroRNA-107, lung cancer, IKBKG, parthenolide, NF-kB, combination effect. advanced NSCLC include radiotherapy and chemotherapy. However, insufficient clinical efficacy has been found due to the low response rate, high rate of recurrence, and drug resistance $(3,4)$. A better understanding of the molecular pathogenesis of NSCLC is required in order to develop novel treatment strategies to improve treatment efficacy.

MicroRNAs (miRs) are small non-coding RNAs that negatively control expression of their target gene via inhibition of translation or degradation of mRNA by binding to the 3' untranslated region (UTR) of the mRNA $(5,6)$. miRs are expressed in a cell- and tissue-specific manner and are differentially expressed in many solid tumors creating a unique signature for each tumor type (7-9). miRs function either as oncogenes or tumor suppressors depending upon their expression level and target genes. For example, miR-145 is down-regulated in several types of cancer, including lung cancer and inhibits cell proliferation of lung adenocarcinoma cells by targeting epidermal growth factor receptor $(E G F R)$ (10). In addition, miRs which can regulate pharmacogenes serve as predictive biomarkers of individual variations in drug response (11).

Nuclear factor kappa-light-chain-enhancer of activated $\mathrm{B}$-cells (NF-kB), which regulates a large number of genes involved in various cellular processes, is constitutively activated in lung cancer and is associated with a poor prognosis (12). Inhibitor of NF-kB kinase subunit gamma (IKBKG, also known as IKK $\gamma$ and NEMO) is a regulatory subunit of the IKB kinase (IKK) core complex required for the activation of NF-KB (13). An inhibition of IKBKG expression using antisense was demonstrated to prevent tumor necrosis factor (TNF)-induced NF-kB activation (14). Targeting NF- $\mathrm{KB}$ has been considered as a potential cancer therapeutic strategy. For example, parthenolide, an inhibitor of NF- $\mathrm{kB}$, has been demonstrated to suppress tumor growth in vitro and in vivo (15). Parthenolide is also known to 
sensitize tumor cells to radiotherapy and conventional anticancer drugs, such as paclitaxel and cisplatin $(16,17)$.

In the present study, we investigated the effects of $m i R-107$ on cell proliferation, target gene expression, and combinatorial effects with parthenolide to elucidate the mechanisms by which miR-107 functions in regulating NSCLC cells.

\section{Materials and Methods}

Cell culture and transfection. A human lung cancer cell line, A549, was obtained from the Korea Cell Line Bank (Seoul, Republic of Korea). Cells were maintained in Dulbecco's modified Eagle's medium (DMEM) supplemented with $10 \%$ fetal bovine serum (FBS) in a humidified $5 \%(\mathrm{v} / \mathrm{v}) \mathrm{CO}_{2}$ atmosphere at $37^{\circ} \mathrm{C}$. For miR transfection, cells were exposed to $50 \mathrm{nM}$ of $\mathrm{miR}$ mimics, specifically miR-control or miR-107 (GE Healthcare, Chicago, IL, USA) using siRNA transfection reagent (RNAiMAX; Invitrogen, Carlsbad, CA, USA) and Opti-MEM medium (Invitrogen) as previously described (18).

Cell proliferation assay. Cells were transfected with $\mathrm{miR}$ mimics (miR-control or miR-107) or exposed to parthenolide at $0.5,1,2.5$, and $5 \mu \mathrm{M}$ for $72 \mathrm{~h}$ under cell culture conditions. Following treatment, cells were then fixed with $10 \%$ trichloroacetic acid and stained with $0.4 \%$ sulforhodamine B (SRB) for 30 mins. Protein-bound dye was extracted with $10 \mathrm{mM}$ Tris base solution, and the optical absorbance of each well was measured at $565 \mathrm{~nm}$. For bromodeoxyuridine (BrdU) staining to detect proliferation, BrdU incorporation assay was performed as previously described (19). Cells were treated with 10 $\mu \mathrm{M} \mathrm{BrdU}$ for $1 \mathrm{~h}$ and then the media were renewed and cells incubated for an additional hour before fixing in methanol at $-20^{\circ} \mathrm{C}$ for 10 mins. Antigen retrieval was performed at $70^{\circ} \mathrm{C}$ in formamide retrieval solution for 30 mins. After blocking in phosphate-buffered saline containing $0.01 \%$ bovine serum albumin and $0.01 \%$ Tween- 20 , slides were incubated for 30 mins with BrdU monoclonal antibody (Developmental Studies Hybridoma Bank, Iowa City, IA, USA). After washing, slides were incubated with Alexa 555-linked secondary antimouse IgG (Invitrogen).

Clonogenic assay. A549 cells transfected with miR-control or miR107 were harvested and plated in DMEM with and without parthenolide treatment $(1.5,2$, and $2.5 \mu \mathrm{M})$ at 5,000-6,000 cells per $100 \mathrm{~mm}$ dish. Two weeks later, the cells were fixed with methanol and stained with crystal violet.

Real time quantitative polymerase chain reaction (PCR). Total RNA was isolated by miRNeasy kit (Qiagen, Hilden, Germany) according to the manufacturer's instructions. To detect mRNA levels, cDNA was prepared using SuperScript III reverse transcription kit (Invitrogen). Real time qPCR was performed on an AriaMx realtime PCR system (Agilent Technologies, Santa Clara, CA, USA) using the Power SYBR Green Master Mix (Applied Biosystems, Foster City, CA, USA) according to the manufacturer's instructions. Primer sequences used in this study were designed by GENOTECH (Daejeon, Republic of Korea). Primer sequences were as follows: inhibitor of nuclear factor kappa B kinase subunit gamma (IKBKG), forward 5'-TGC CTG GAG GAG AAT CAA-3' and reverse 5'CGC AGA ATC TGG TTG CTC-3'; glyceraldehyde-3-phosphate dehydrogenase $(G A P D H)$, forward 5'-GAA GGT GAA GGT CGG
AGT C-3' and reverse 5'-GAA GAT GGT GAT GGG ATT TC-3'. Luciferase reporter constructs and assay. The three prime untranslated regions (3'UTR) of miR-107 predicted targets were PCR amplified using human genomic DNA as a template and a LongAmp ${ }^{\text {TM }}$ TaqDNA Polymerase (New England BioLabs, Ipswich, MA, USA). The PCR amplicons were ligated into the psiCHECK-2 vector (Promega, Madison, WI, USA). The mutant reporter constructs harboring mutation of the first three nucleotides of the seed-match sequence were constructed using the QuikChange site-directed mutagenesis kit (Stratagene, San Diego, CA, USA). The interaction of the target genes with $m i R-107$ was screened using the psiCHECK-2 vector harboring a wild or mutant 3' UTR. Constructs were co-transfected with either miR-control or miR-107 into cells. Twenty-four hours after transfection, cell lysates were used to measure both firefly and renilla luciferase activities using the Dual-Luciferase ${ }^{\circledR}$ Reporter Assay System (Promega) following the manufacturer's instructions. Primer sequences used in this study were designed by GENOTECH. Primer sequences were as follows: BCL2-interacting protein 3 (BNIP3), forward 5'-CTG CCT CGA GTA TAT TGG AAG GCG TCT GAC-3' and reverse 5'ATT AGC GGC CGC GTG TGA TAT ATA AGG CCA CAA-3'; cathepsin D (CTSD), forward 5'-CTG CCT CGA GCA CAG AAA CAG AGG AGA GTC-3' and reverse 5'-ATT AGC GGC CGC ACT GTA TTT CCA TGT CAG CT-3'; caspase 3 (CASP3), forward 5'CTG CCT CGA GTC TTG GCG AAA TTC AAA GGA-3' and reverse 5'-ATT AGC GGC CGC AAT TGT CAC ATA GAA ACA CAC T-3'; regulator of calcineurin 1 (RCAN1), forward 5'-CTG CCT CGA GCA AAA ATT ATC CAG ACC AGG AG-3' and reverse 5'ATT AGC GGC CGC TTT TCT GTA AGG AGC ATA CT-3'; zyxin $(Z Y X)$, forward 5'-CTG CCT CGA GCT TCA GAC CGC AGT CCA T-3' and reverse 5'-ATT AGC GGC CGC TCT GGA AAA CAA GGT TTT TTT TTA TTT GTA G-3'; $I K B K G$, forward 5'-CTG CCT CGA GAT GTC ATG GAG TGC ATT GAG-3' and reverse 5'-ATT AGC GGC CGC TAC TTC TCT ATT GGG GTC AC-3'; protein phosphatase 1 catalytic subunit alpha (PPP1CA), forward 5'-CTG CCT CGA GCC AAA GCC AAG AAA TAG CC-3' and reverse 5'ATT AGC GGC CGC TTT ATT CAA GAG ACC AGA TGG GT-3'; $P P P 1 C C$, forward 5'-CTG CCT CGA GAT GTC GTT TTG ACA CTG CCT-3' and reverse 5'-ATT AGC GGC CGC GTT CCA CCT CAT CAC TCT ATT-3'; PPP3CA, forward 5'-CTG CCT CGA GCC ACT TCC TGT TCA CTT TTT-3' and reverse 5'-ATT AGC GGC CGC TTA ATG TCA AAT GTT TAT TTC TAT GCC ATA-3’.

Protein extraction and immunoblotting. Proteins were extracted with RIPA cell lysis buffer (Thermo Fisher Scientific, Waltham, MA, USA), and phosphatase/protease inhibitor cocktail (Thermo Fisher Scientific). 4-20\% Tris-HCl gradient gels (Bio-Rad Laboratories, Hercules, CA, USA) were used to separate proteins. Blotting was performed for IKBKG (DA10-12) (Cell Signaling Technology, Danvers, MA, USA) and GAPDH (Santa Cruz Biotechnology, Inc., Dallas, TX, USA).

Data analysis. The $\mathrm{IC}_{50}$ was defined as the drug concentration at which cell viability was equal to $50 \%$ that of the control without drug, and calculated using Eq. 1 and Eq. 2:

$$
\begin{gathered}
\text { Cell viability }(\%)=\left(\frac{\text { Mean absorbance of treated cells }}{\text { Mean absorbance of control cells }}\right) \times 100(\text { Eq. 1) } \\
\text { Cell viability }(\%)=(100-\mathrm{R}) \times\left(1-\frac{[D]^{m}}{K_{d}^{m}+[D]^{m}}\right) \times 100(\text { Eq. 2) }
\end{gathered}
$$


A

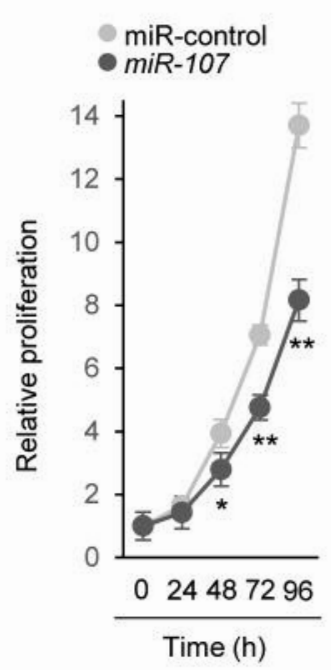

B

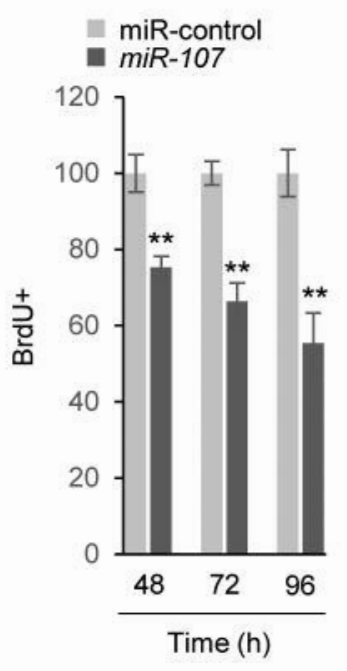

C
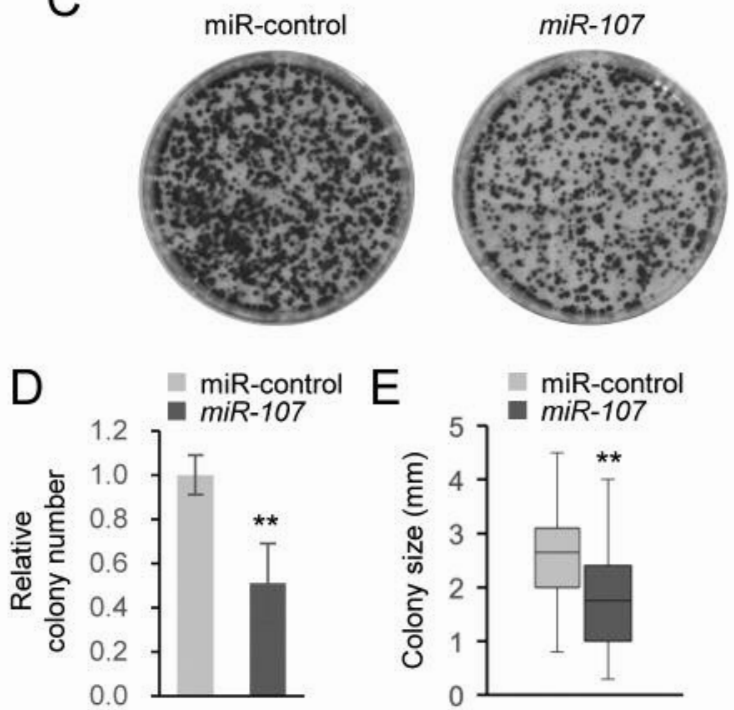

Figure 1. miR-107 regulates the proliferative status of A549 cells. A: Cell growth in A549 cells transfected with miR-control or miR-107 was analyzed by the SRB assay. Data are shown as means $\pm S D(N=3)$. B: Bromodeoxyuridine (BrdU) analysis of A549 cells transfected with miR-control or miR107 showing cells in the S-phase of DNA synthesis. C: Representative images of a clonogenic assay of A549 cells following transfection of miRcontrol or miR-107. Quantification of the number $(D)$ and size $(E)$ of colonies induced by overexpression of miR-control or miR-107 in A549 cells. Significantly different at $* p<0.05$ and $* * p<0.01$. Data are means $\pm S D$.

where $D$ is the drug concentration, $m$ is the Hill-type coefficient, $R$ is the residual unaffected fraction (the resistance fraction), and $K d$ is the concentration of drug that produces a $50 \%$ reduction of the drug's maximum effect (100-R) (20). The effect of combination of drugs at fixed concentrations was analyzed by comparing experimental data to the reference additivity values calculated using Bliss independence model. Values of the ratio of the experimental survival rate to the reference value between 0.8 and 1.2 was defined as additive, $\leq 0.8$ as synergistic, and $\geq 1.2$ as antagonistic (20).

Analysis of publicly available datasets. RNA sequencing data of 525 lung cancer samples were downloaded from The Cancer Genome Atlas database to analyze the potential correlation between $m i R-107$ and $I K B K G$. The significance of the inverse association between $m i R-107$ and $I K B K G$ was assessed by Pearson's chi-squared test with Yates' continuity correction.

Statistical analysis. An unpaired $t$-test and analysis of variance were used to determine statistical significance. The data are shown as means $\pm \mathrm{SD}$. The differences were considered significant for $p$-values of less than 0.05 . All experiments were replicated at least three times.

\section{Results}

miR-107 inhibits the proliferation of A549 cells. Our previous work demonstrated that miR-107 negatively regulates family of retrovirus-associated DNA sequences (RAS), src-family kinase (SRC), and neural precursor cell expressed, developmentally down-regulated 9 (NEDD9) signaling pathways involved in proliferation of epithelial cells $(18,19)$. To explore the possibility that $m i R-107$ alters the proliferative status of lung cancer cells, we analyzed the effects of increasing miR-107 in A549 cells. A significant reduction in cell growth was noted for A549 cells up to 96 $\mathrm{h}$ after transfection of $m i R-107$ (Figure 1A). This reduction of cell proliferation was also evidenced by a significant decrease in the number of A549 cells in the S-phase of cell cycle (Figure 1B). To determine the effect of $m i R-107$ on tumor-initiating capacity, we utilized a clonogenic assay for A549 cells. The miR-107-transfected A549 cells gave rise to significantly fewer colonies than the miR-control-transfected cells (Figure 1C and D). Indeed, miR-107 treatment of A549 cells also resulted in an overall decrease of colony size (Figure 1E). Such results demonstrate that miR-107 contributes to a malignant phenotype by regulating lung cancer cell proliferation.

miR-107 directly targets IKBKG. In order to understand how miR-107 functions in regulating cell proliferation, we used bioinformatic predictions and cell-based luciferase assays to find a target gene. The TargetScan algorithm was used to search for potential targets that have a $m i R-107$ binding site within the 3' UTR. Of nine potential predicted targets, only $I K B K G$ was shown to be a direct target of miR-107 in A549 cells (Figure 2A). Since two $m i R-107$ binding sites were predicted in the 3'UTR of IKBKG (ENST00000393549), mutations in the seedmatch sequence were introduced to determine the functional 
A

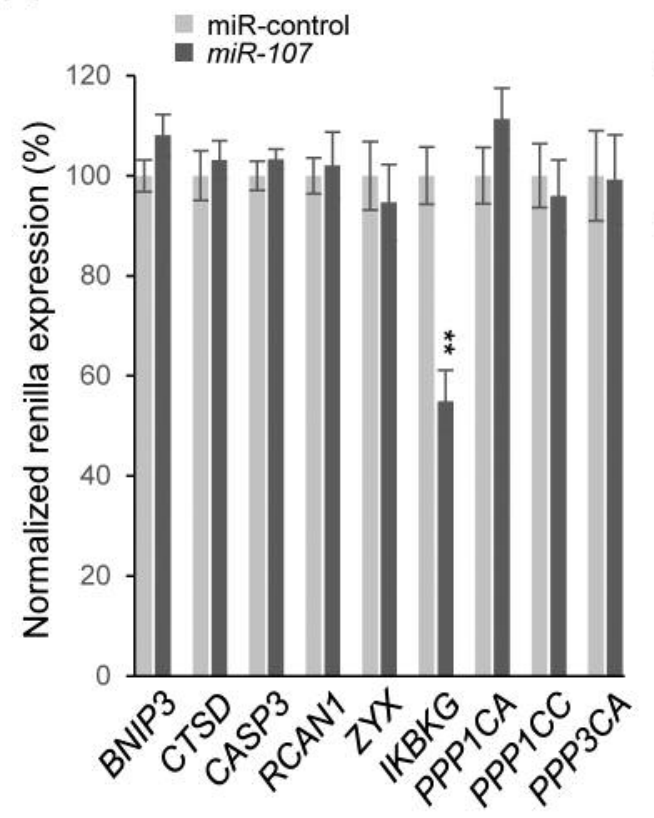

B

$1^{\text {st }}$ binding site

IKBKG 3'UTR (mutant, MUT1) 5' UUGUCUGCUCACGGGUGCCCAAC 3' IKBKG 3'UTR (wild type) 5' UUGUCUGCUCACGGGUGCUGCAC 3' hsa-miR-107 3' CUAUCGGGACAUGUUACGACGA 5'

$2^{\text {nd }}$ binding site

IKBKG 3'UTR (mutant, MUT2) 5' CAGCGGCCCUUGCA-AUGCCCAC 3', IKBKG 3'UTR (wild type) 5' CAGCGGCCCUUGCA--AUGCUGCC 3' hsa-miR-107 3' ACUAUCGGGACAUGUUACGACGA 5'

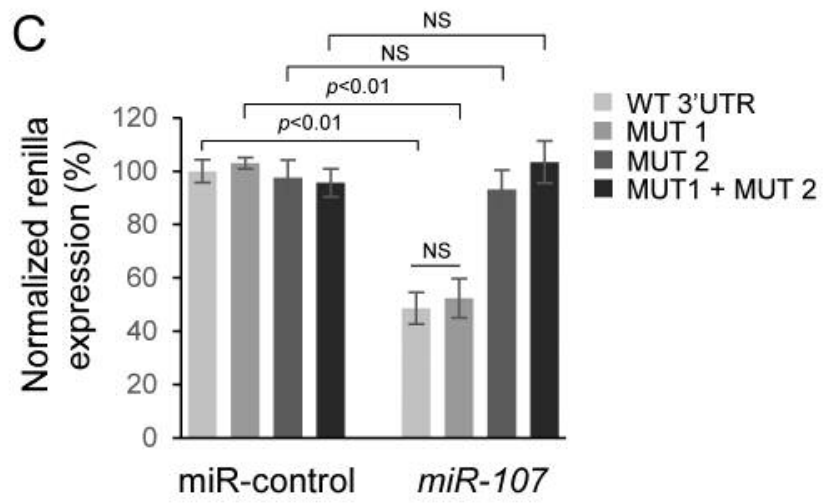

Figure 2. miR-107 targets inhibitor of nuclear factor kappa B kinase subunit gamma (IKBKG), an essential modulator of nuclear factor kappa B $(N F-k B)$. A: Screening of predicted target genes of miR-107 using the psiCHECK-2 constructs harboring a 3'untranslated region (UTR) of each gene. Predicted target genes were selected based on the effects on cell proliferation. The construct was co-transfected with either miR-control or miR-107 into A549 cells. Twenty-four hours after transfection, cell lysates were used to measure both firefly and renilla luciferase activities using the dual-luciferase reporter assay system. B: A schematic diagram of miR-107 binding sites in the 3'UTR region of IKBKG mRNA. Mutant reporter constructs were generated at the first three nucleotides of the seed-match sequence. C: Luciferase assays to test the interaction of IKBKG mRNA with miR-107 using the psiCHECK-2 vector harboring a wild-type or mutant 3' UTR of IKBKG. Constructs were co-transfected with either miRcontrol or miR-107 into A549 cells. **Significantly different at $p<0.01 ; N S$, not significant. Data are means $\pm S D$.

target site of $m i R-107$ (Figure 2B). Mutation of the first binding site (MUT1) led to no significant change when compared with wild-type constructs in $m i R$-107-overexpressing cells. In contrast, luciferase activity in A549 cells transfected with vectors harboring the mutation at the second binding site (MUT2 and MUT1+MUT2) was completely rescued, suggesting that the second binding site participates in the interaction between $m i R-107$ and $I K B K G$ mRNA (Figure 2C).

Endogenous IKBKG expression is down-regulated in miR-107overexpressing cells. To further experimentally validate if the $I K B K G$ mRNA indeed interacts with miR-107, real time qPCR analysis was first performed in A549 cells following overexpression of either miR-control or miR-107. We observed that the IKBKG mRNA level was reduced by approximately $24 \%$ and $29 \%$ at 12 and $24 \mathrm{~h}$, respectively, after overexpression of miR-107 in A549 cells (Figure 3A). A significant reduction in IKBKG protein was also noted in A549 cells transfected with $m i R-107$ compared to miR-control (Figure 3B), suggesting that $I K B K G$ expression can be downregulated by both mRNA degradation and translational repression. To analyze the potential correlation between $m i R$ 107 and its target gene, $I K B K G$, we collected miR and mRNA gene expression data on 525 lung cancer samples from The Cancer Genome Atlas database. IKBKG mRNA exhibited significantly lower expression levels in the lung cancer samples with high $m i R-107$ levels, respectively $(\mathrm{X}=5.26, \mathrm{df}=1$, $p=0.02$ ), confirming our data that $I K B K G$ is regulated by $m i R$ 107 (Figure 3C).

miR-107 sensitizes A549 cells to parthenolide. Parthenolide, a natural sesquiterpene lactone, has been reported to exhibit antiproliferative effects on various human cancer cells, such as pancreatic carcinoma, breast cancer, and NSCLC $(15,21$, 22). As such, we first evaluated the antiproliferative activity of parthenolide treatment on A549 cells. Cells were exposed to parthenolide up to $5 \mu \mathrm{M}$ for $72 \mathrm{~h}$, and we found that parthenolide treatment had strong antiproliferative effects with a relatively low $\mathrm{IC}_{50}$ value $(2.53 \mu \mathrm{M})$ in A549 cells (Figure 4A). A previous study has demonstrated that the antiproliferative activity of parthenolide could be enhanced by co-treatment with sulindac via cooperatively inhibiting 
A

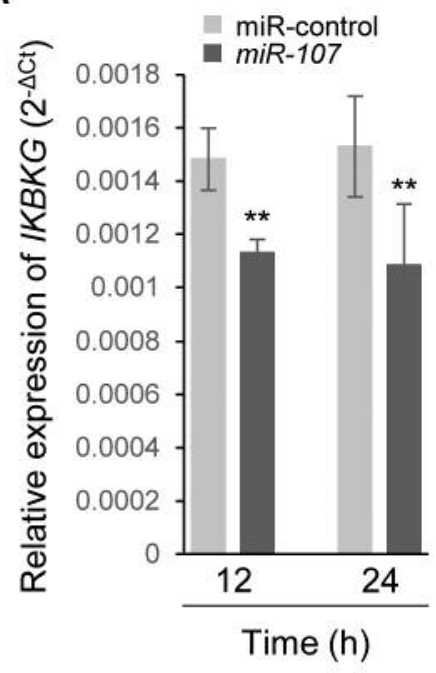

B

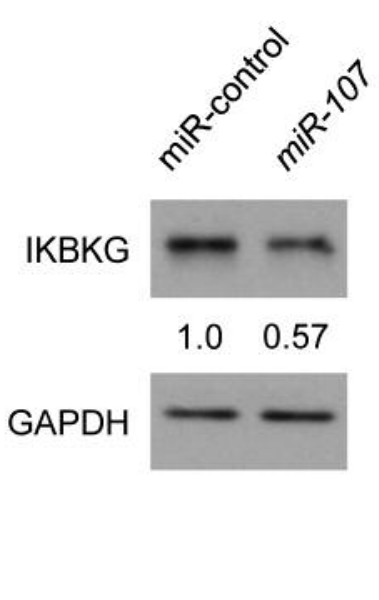

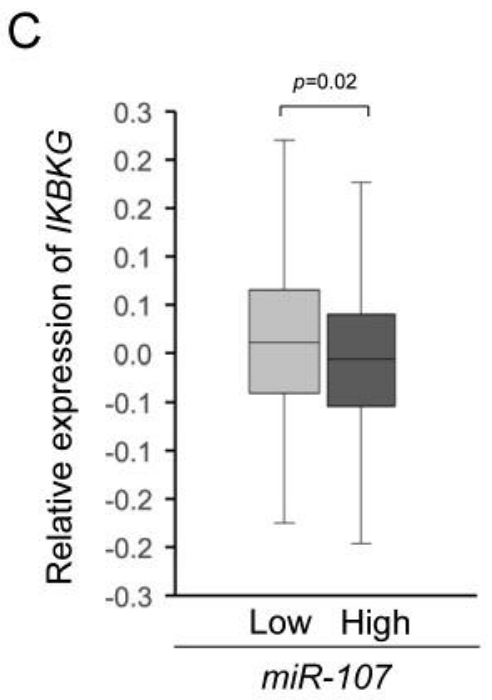

Figure 3. Inhibitor of nuclear factor kappa B kinase subunit gamma (IKBKG) is down-regulated in miR-107-overexpressing cells. A: Real-time quantitative polymerase chain reaction analyses of IKBKG $\mathrm{mRNA}$ levels in A549 cells transfected with miR-control or miR-107 at $50 \mathrm{nM}$. The data are the means $\pm S D$ for three independent experiments. B: A549 cells were transfected with either miR-control or miR-107 at $50 \mathrm{nM}$ for $48 \mathrm{~h}$, and then protein lysates were isolated and subjected to western blot analysis of endogenous IKBKG. Glyceraldehyde-3-phosphate dehydrogenase $(G A P D H)$ served as a loading control and relative expressions are indicated. C: RNA sequencing data of 525 lung cancer samples were downloaded from The Cancer Genome Atlas data portal. The statistical significance of the association between miR-107 and IKBKG was assessed by Pearson's Chi-squared test with Yates' continuity correction. ${ }^{* *}$ Significantly different at $p<0.01$. Data are means $\pm S D$.

NF-kB pathway (21). Therefore, we next sought to investigate whether the antiproliferative effect of parthenolide is improved by $m i R-107$ which targets $I K B K G$. The effect of parthenolide was evaluated $72 \mathrm{~h}$ after incubation, which took place $24 \mathrm{~h}$ after transfection of miRcontrol or miR-107 in A549 cells (Figure 4B). Treatment with parthenolide alone at $1.5,2$ and $2.5 \mu \mathrm{M}$ resulted in about $96.8 \%, 61.3 \%$, and $49.9 \%$ survival, respectively, when compared to the vehicle-treated cells transfected with miRcontrol (Figure 4B). The combination of parthenolide with miR-107 induced strong synergistic antiproliferative effects in A549 cells. Exposure to 1.5, 2 and $2.5 \mu \mathrm{M}$ of parthenolide produced $63 \%, 26.9 \%$, and $27.8 \%$ survival, respectively, when compared with the vehicle treated cells overexpressing miR-107 (Figure 4B). Based on our findings, we further examined the combination effect of miR-107 and parthenolide using a clonogenic assay. The results clearly showed that treatment with parthenolide in A549 cells overexpressing $m i R-107$ gave rise to significantly fewer colonies than the combination of miR-control and parthenolide (Figure 4C-E). In addition, real-time qPCR analysis showed that treatment with parthenolide dramatically reduced the mRNA level of $C D K 2$, a target of NF-kB (23), when combined with miR-107 in A549 cells (Figure 4F). No statistically significant differences were observed for $C D K 2$ mRNA level in cells treated with miRcontrol and parthenolide.

\section{Discussion}

Here we furnish evidence that miR-107 acts as a tumorsuppressive non-coding RNA showing the short-term and long-term in vitro antiproliferative effects on A549 NSCLC cells. In addition, $m i R-107$ targets $I K B K G$, a critical regulator of NF-kB activation, and miR-107 overexpressing A549 cells are more sensitive to parthenolide.

Various microRNAs (miRs) are aberrantly expressed in NSCLC and other types of cancer. We recently reported that several miR, including $m i R-337-3 p$ are dysregulated in pancreatic ductal adenocarcinoma and they directly regulate the expression of executioner caspases-3 and caspase-7 in PANC1 cells (8). Another study indicates that $m i R-595$ targets ATPbinding cassette subfamily B member 1 ( $A B C B 1$, also called P-glycoprotein), thereby sensitizing ovarian cancer cells to cisplatin (24). Differentially expressed miR in NSCLC include $m i R-21, m i R-221, m i R-148$, and $m i R-107$ (25). Accumulating evidence has shown that the oncogenic or tumor-suppressor role of miR-107 depends on the type of cancer. By targeting forkhead box O1 (FOXO1), death-associated protein kinase 1 $(D A P K)$, and kruppel-like factor 4 (KLF4), miR-107 promotes the cell proliferation and metastasis of gastric and colorectal cancer $(26,27)$. In contrast, restriction of glioma cell invasion is induced by miR-107 via regulation of NOTCH2 expression (28). With respect to NSCLC, miR-107 is significantly downregulated in lung cancer compared to normal tissues (29). 
A

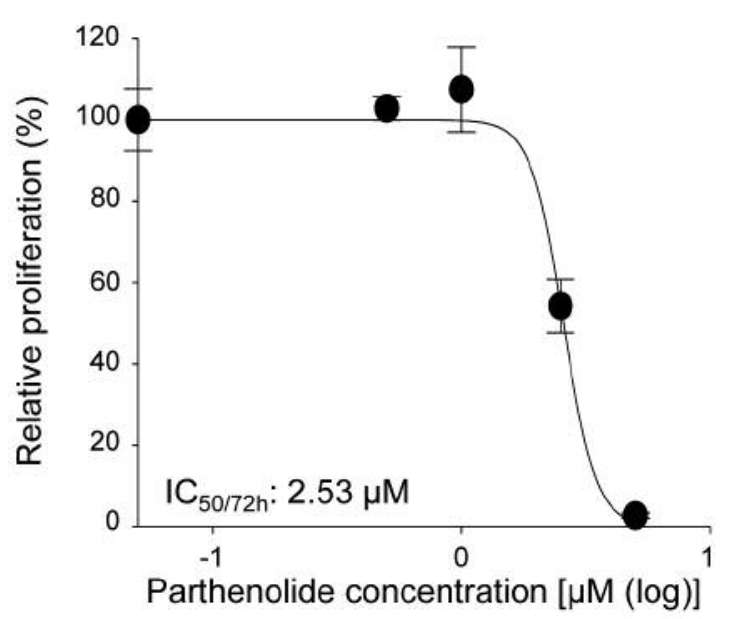

C
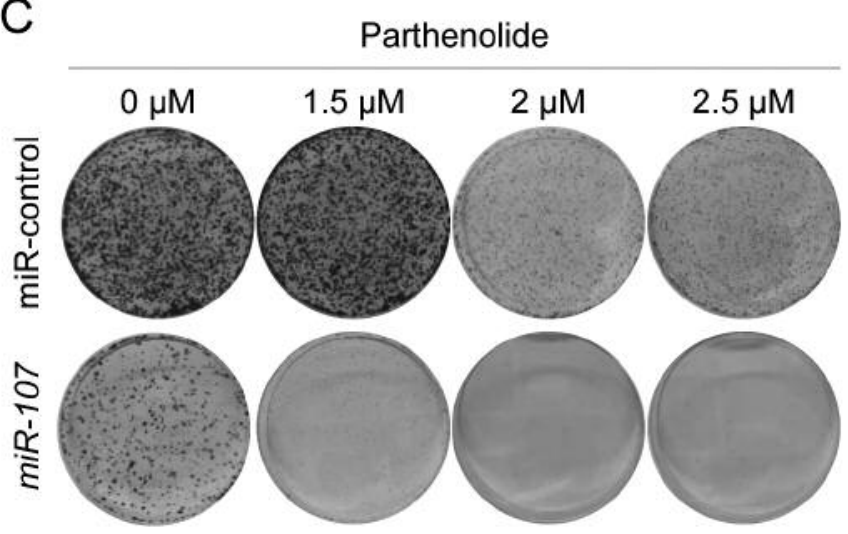

E

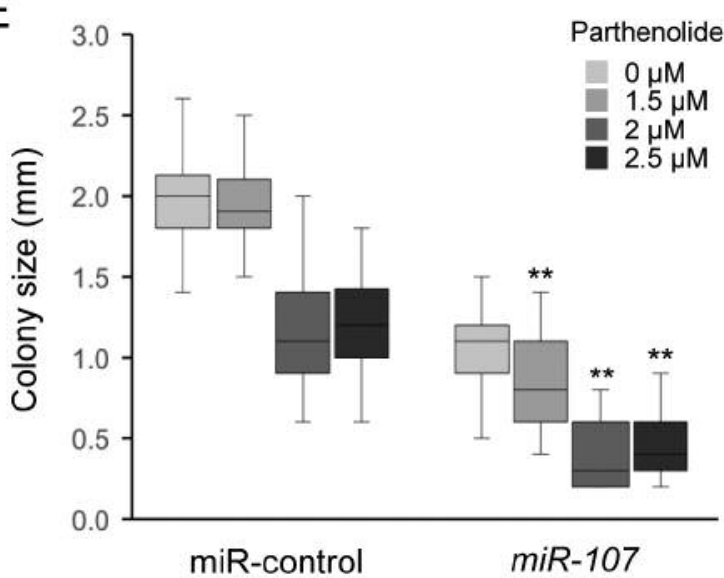

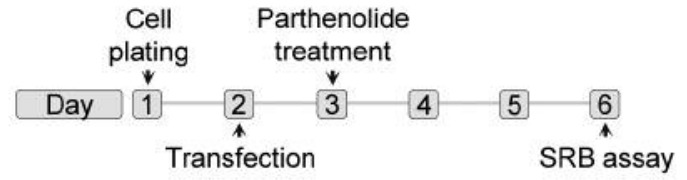

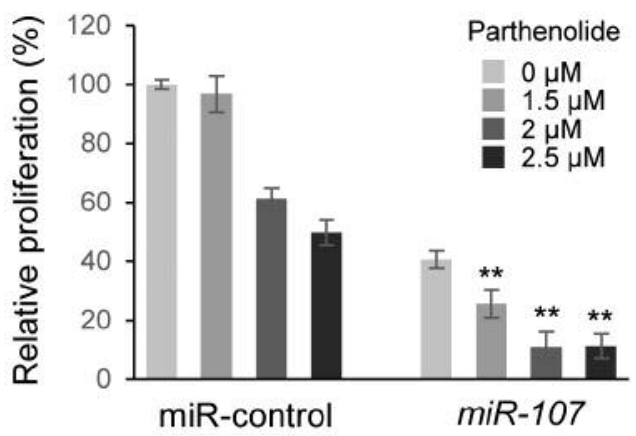

$\mathrm{D}$

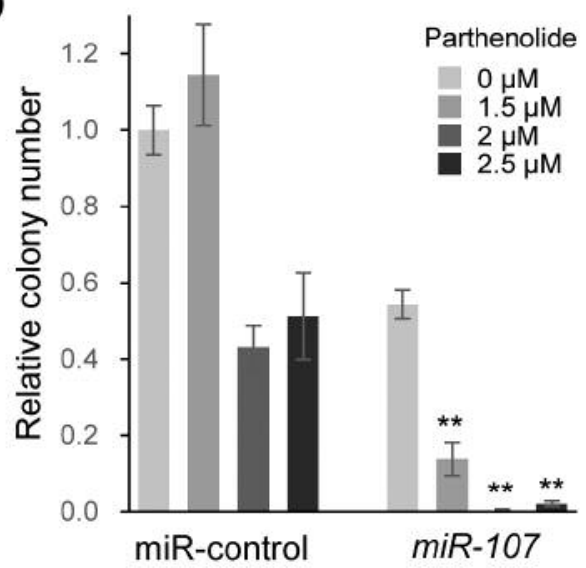

$\mathrm{F}$

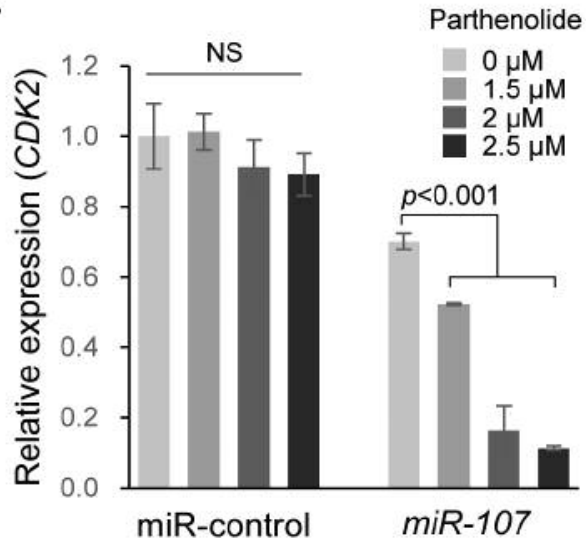

Figure 4. miR-107 sensitizes A549 cells to the effects of parthenolide. A: A representative dose-response curve of A549 cells treated with different concentrations of parthenolide. Cell viability was assessed following $72 \mathrm{~h}$ continuous exposure. B: Cells were treated with parthenolide at the indicated concentrations following transfection of A549 cells with either miR-control or miR-107. Survival rates were determined using the sulforhodamine B assay $72 \mathrm{~h}$ after treatment of parthenolide. C: Representative images of a clonogenic assay of A549 cells following a combination of microRNA mimics (miRcontrol or miR-107) with parthenolide. Quantification of the number $(D)$ and size $(E)$ of colonies induced by a combination of microRNA mimics (miRcontrol or miR-107) with parthenolide. F: Treatment conditions were the same as in (B) and $(C)$ and the expression level of cyclin-dependent kinase 2 (CDK2) mRNA in A549 cells was determined by real-time quantitative polymerase chain reaction. ${ }^{* *}$ Indicates synergistic effects. Data are means $\pm S D$. 
Consistent with the decrease in cell proliferation and BrdU labeling in $m i R$-107-overexpressing A549 cells (Figure 1), it was demonstrated that miR-107 arrests H1299 NSCLC cells at the G1 cell-cycle phase (29). In addition, $m i R-107$ has been reported to augment the effects of paclitaxel on NSCLC via modulation of BCL2-like 2 (BCL2L2) expression (30). We demonstrate enhanced parthenolide sensitivity in conjunction with miR-107 along with the notable inhibition of $C D K 2$ expression (Figure 4). These findings indicate the possibility that $m i R-107$ may contribute to improve therapeutic efficacy of various anticancer drugs for NSCLC.

The proliferation of lung cancer cells can be promoted through NF-kB activation by an oncogene, tripartite motifcontaining 29 (TRIM29) (31). NF-KB is activated in lung cancer cells by small GTPase RAC1. Pharmacological and genetic inhibition of RAC1 strongly blocks cell proliferation along with the inhibition of NF-kB activity (32). NF-kB has also been reported to associate with the acquisition of chemoresistance in cancer cells to various anticancer agents. NF- $\mathrm{kB}$ activation is involved in the development of drug resistance to 5-fluorouracil in human colon cancer cells (33). The inhibition of NF-kB sensitizes colon cancer cells to daunomycin by down-regulating P-glycoprotein expression (34). Recently, it was demonstrated that targeted inhibition of NF-kB translocation using dehydroxymethylepoxyquinomicin has a promising effect on cisplatin-resistant NSCLC cells (35). Consistent with the involvement of NF-kB in chemoresistance, it was demonstrated that knockdown of $I K B K G$ using a genetic approach significantly intensifies the efficacy of radiation therapy in lung cancer cells (36). Therefore, our observation that miR-107 targets $I K B K G$ suggests the possibility that $m i R-107$ may be able to ameliorate anticancer drug-induced chemoresistance and effectively inhibit the proliferation of chemoresistant tumor cells.

Our findings not only represent one of the examples of how miR can influence cancer cell proliferation, but also suggest that by regulating a NF-kB modulator, IKBKG, miR107 potentiates the efficacy of parthenolide against NSCLC cells. Since unprecedented advantages of combination treatment of miR with anticancer drugs have been suggested (37), we believe that $m i R-107$, in conjunction with parthenolide or other anticancer agents, may represent a novel means of combination treatment of NSCLC.

\section{Conflicts of Interest}

The Authors declare that they have no competing interests in regard to this study.

\section{Acknowledgements}

This study was supported by a grant from the Basic Science Research Program through the National Research Foundation of Korea (NRF) funded by the Ministry of Education (2017R1D1A3B03035662), and Hallym University Research Fund, 2017(HRF-201703-003).

\section{References}

1 Siegel RL, Miller KD and Jemal A: Cancer statistics, 2017. CA Cancer J Clin 67: 7-30, 2017.

2 Zarogoulidis K, Zarogoulidis P, Darwiche K, Boutsikou E, Machairiotis N, Tsakiridis K, Katsikogiannis N, Kougioumtzi I, Karapantzos I, Huang H and Spyratos D: Treatment of non-small cell lung cancer (NSCLC). J Thorac Dis 5(Suppl 4): S389-396, 2013.

3 Tsvetkova E and Goss GD: Drug resistance and its significance for treatment decisions in non-small-cell lung cancer. Curr Oncol 19: S45-51, 2012.

4 Wangari-Talbot $\mathrm{J}$ and Hopper-Borge E: Drug resistance mechanisms in non-small cell lung carcinoma. J Can Res Updates 2: 265-282, 2013.

5 Yates LA, Norbury CJ and Gilbert RJ: The long and short of microRNA. Cell 153: 516-519, 2013.

6 Macfarlane LA and Murphy PR: MicroRNA: Biogenesis, function and role in cancer. Curr Genomics 11: 537-561, 2010.

7 Lu J, Getz G, Miska EA, Alvarez-Saavedra E, Lamb J, Peck D, Sweet-Cordero A, Ebert BL, Mak RH, Ferrando AA, Downing JR, Jacks T, Horvitz HR and Golub TR: MicroRNA expression profiles classify human cancers. Nature 435: 834-838, 2005.

8 Park JK, Doseff AI and Schmittgen TD: MicroRNAs targeting caspase-3 and -7 in panc-1 cells. Int J Mol Sci 19, 2018.

9 Hoefert JE, Bjerke GA, Wang D and Yi R: The microRNA-200 family coordinately regulates cell adhesion and proliferation in hair morphogenesis. J Cell Biol 217: 2185-2204, 2018.

10 Cho WC, Chow AS and Au JS: Mir-145 inhibits cell proliferation of human lung adenocarcinoma by targeting egfr and nudt1. RNA Biol 8: 125-131, 2011.

11 Han N, Song YK, Burckart GJ, Ji E, Kim IW and Oh JM: Regulation of pharmacogene expression by microRNA in The Cancer Genome Atlas (TCGA) Research Network. Biomol Ther 25: 482-489, 2017.

12 Cai Z, Tchou-Wong KM and Rom WN: NF-kappaB in lung tumorigenesis. Cancers 3: 4258-4268, 2011.

13 May MJ, D'Acquisto F, Madge LA, Glockner J, Pober JS and Ghosh S: Selective inhibition of nf-kappab activation by a peptide that blocks the interaction of nemo with the ikappab kinase complex. Science 289: 1550-1554, 2000.

14 Rothwarf DM, Zandi E, Natoli G and Karin M: IKK-gamma is an essential regulatory subunit of the IkappaB kinase complex. Nature 395: 297-300, 1998.

15 Lin M, Bi H, Yan Y, Huang W, Zhang G, Zhang G, Tang S, Liu Y, Zhang L, Ma J and Zhang J: Parthenolide suppresses nonsmall cell lung cancer GLC-82 cells growth via BRAF/MAPK/ERK pathway. Oncotarget 8: 23436-23447, 2017.

16 Sohma I, Fujiwara Y, Sugita Y, Yoshioka A, Shirakawa M, Moon JH, Takiguchi S, Miyata H, Yamasaki M, Mori M and Doki Y: Parthenolide, an NF-kappaB inhibitor, suppresses tumor growth and enhances response to chemotherapy in gastric cancer. Cancer Genomics Proteomics 8: 39-47, 2011.

17 Morel KL, Ormsby RJ, Bezak E, Sweeney CJ and Sykes PJ: Parthenolide selectively sensitizes prostate tumor tissue to radiotherapy while protecting healthy tissues in vivo. Radiat Res 187: 501-512, 2017.

18 Park JK, Peng H, Katsnelson J, Yang W, Kaplan N, Dong Y, Rappoport JZ, He C and Lavker RM: MicroRNAs-103/107 coordinately regulate macropinocytosis and autophagy. J Cell Biol 215: 667-685, 2016. 
19 Peng H, Park JK, Katsnelson J, Kaplan N, Yang W, Getsios S and Lavker RM: MicroRNA-103/107 family regulates multiple epithelial stem cell characteristics. Stem Cells 33: 1642-1656, 2015.

20 Roell KR, Reif DM and Motsinger-Reif AA: An introduction to terminology and methodology of chemical synergy-perspectives from across disciplines. Front Pharmacol 8: 158, 2017.

21 Yip-Schneider MT, Nakshatri H, Sweeney CJ, Marshall MS, Wiebke EA and Schmidt CM: Parthenolide and sulindac cooperate to mediate growth suppression and inhibit the nuclear factor-kappa B pathway in pancreatic carcinoma cells. Mol Cancer Ther 4: 587-594, 2005.

22 D'Anneo A, Carlisi D, Lauricella M, Puleio R, Martinez R, Di Bella S, Di Marco P, Emanuele S, Di Fiore R, Guercio A, Vento $\mathrm{R}$ and Tesoriere G: Parthenolide generates reactive oxygen species and autophagy in MDA-MB231 cells. A soluble parthenolide analogue inhibits tumour growth and metastasis in a xenograft model of breast cancer. Cell Death Dis 4: e891, 2013.

23 Godwin P, Baird AM, Heavey S, Barr MP, O'Byrne KJ and Gately K: Targeting nuclear factor-kappa B to overcome resistance to chemotherapy. Front Oncol 3: 120, 2013.

24 Tian S, Zhang M, Chen X, Liu Y and Lou G: MicroRNA-595 sensitizes ovarian cancer cells to cisplatin by targeting ABCB1. Oncotarget 7: 87091-87099, 2016.

25 Garofalo M, Romano G, Di Leva G, Nuovo G, Jeon YJ, Ngankeu A, Sun J, Lovat F, Alder H, Condorelli G, Engelman JA, Ono M, Rho JK, Cascione L, Volinia S, Nephew KP and Croce CM: EGFR and met receptor tyrosine kinase-altered microRNA expression induces tumorigenesis and gefitinib resistance in lung cancers. Nat Med 18: 74-82, 2011.

26 Li F, Liu B, Gao Y, Liu Y, Xu Y, Tong W and Zhang A: Upregulation of microRNA-107 induces proliferation in human gastric cancer cells by targeting the transcription factor foxo1. FEBS Lett 588: 538-544, 2014.

27 Chen HY, Lin YM, Chung HC, Lang YD, Lin CJ, Huang J, Wang WC, Lin FM, Chen Z, Huang HD, Shyy JY, Liang JT and Chen RH: Mir-103/107 promote metastasis of colorectal cancer by targeting the metastasis suppressors dapk and klf4. Cancer Res 72: 3631-3641, 2012.

28 Chen L, Chen XR, Zhang R, Li P, Liu Y, Yan K and Jiang XD: MicroRNA-107 inhibits glioma cell migration and invasion by modulating NOTCH2 expression. J Neurooncol 112: 59-66, 2013.
29 Takahashi Y, Forrest AR, Maeno E, Hashimoto T, Daub CO and Yasuda J: mir-107 and mir-185 can induce cell cycle arrest in human non small cell lung cancer cell lines. PLoS One 4: e6677, 2009.

$30 \mathrm{Lu} \mathrm{C}$, Xie Z and Peng Q: MiRNA-107 enhances chemosensitivity to paclitaxel by targeting antiapoptotic factor BCL-w in non small cell lung cancer. Am J Cancer Res 7: 1863-1873, 2017.

31 Tang ZP, Dong QZ, Cui QZ, Papavassiliou P, Wang ED and Wang EH: Ataxia-telangiectasia group D complementing gene (ATDC) promotes lung cancer cell proliferation by activating nfkappab pathway. PLoS One 8: e63676, 2013.

32 Gastonguay A, Berg T, Hauser AD, Schuld N, Lorimer E and Williams CL: The role of rac1 in the regulation of NF-kappaB activity, cell proliferation, and cell migration in non-small cell lung carcinoma. Cancer Biol Ther 13: 647-656, 2012.

33 Korber MI, Staribacher A, Ratzenbock I, Steger G and Mader RM:NF-kappaB-associated pathways in progression of chemoresistance to 5-fluorouracil in an in vitro model of colonic carcinoma. Anticancer Res 36: 1631-1639, 2016.

34 Bentires-Alj M, Barbu V, Fillet M, Chariot A, Relic B, Jacobs N, Gielen J, Merville MP and Bours V: NF-kappaB transcription factor induces drug resistance through MDR1 expression in cancer cells. Oncogene 22: 90-97, 2003.

35 Heavey S, Godwin P, Baird AM, Barr MP, Umezawa K, Cuffe S, Finn SP, O'Byrne KJ and Gately K: Strategic targeting of the PI3K-NF-kappaB axis in cisplatin-resistant NSCLC. Cancer Biol Ther 15: 1367-1377, 2014.

36 Tsolou A, Liousia M, Kalamida D, Pouliliou S, Giatromanolaki A and Koukourakis M: Inhibition of IKK-NFkappaB pathway sensitizes lung cancer cell lines to radiation. Cancer Biol Med 14: 293-301, 2017.

37 Dai $\mathrm{X}$ and Tan C: Combination of microRNA therapeutics with small-molecule anticancer drugs: Mechanism of action and codelivery nanocarriers. Adv Drug Deliv Rev 81: 184-197, 2015.

Received September 18, 2018

Revised October 4, 2018

Accepted October 8, 2018 